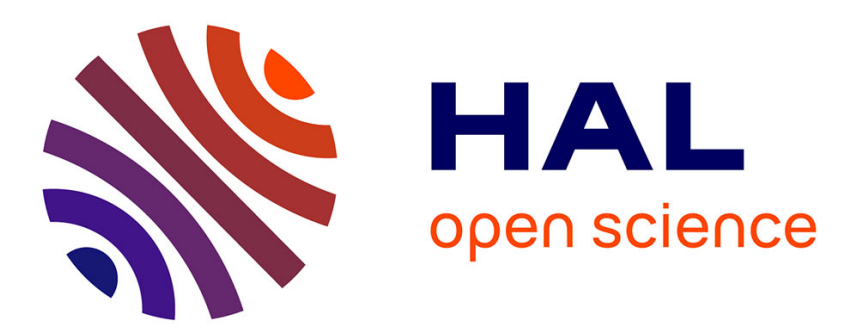

\title{
Intact monoclonal antibodies separation and analysis by sheathless capillary electrophoresis-mass spectrometry
} Jeremie Giorgetti, Antony Lechner, Elise del Nero, Alain Beck, Emmanuelle Leize-Wagner, Yannis-Nicolas François

\section{- To cite this version:}

Jeremie Giorgetti, Antony Lechner, Elise del Nero, Alain Beck, Emmanuelle Leize-Wagner, et al.. Intact monoclonal antibodies separation and analysis by sheathless capillary electrophoresismass spectrometry. European Journal of Mass Spectrometry, 2019, 25 (3), pp.324-332. 10.1177/1469066718807798 . hal-01981306

\author{
HAL Id: hal-01981306 \\ https://hal.science/hal-01981306
}

Submitted on 14 Jan 2019

HAL is a multi-disciplinary open access archive for the deposit and dissemination of scientific research documents, whether they are published or not. The documents may come from teaching and research institutions in France or abroad, or from public or private research centers.
L'archive ouverte pluridisciplinaire HAL, est destinée au dépôt et à la diffusion de documents scientifiques de niveau recherche, publiés ou non, émanant des établissements d'enseignement et de recherche français ou étrangers, des laboratoires publics ou privés. 


\title{
Intact monoclonal antibodies separation and analysis by sheathless capillary electrophoresis-mass spectrometry
}

\author{
Jérémie Giorgetti ${ }^{1}$, Antony Lechner ${ }^{1}$, Elise Del Nero ${ }^{1}$, Alain Beck ${ }^{2}$, Emmanuelle Leize- $^{-}$ \\ Wagner $^{1}$, Yannis-Nicolas François ${ }^{1}$
}

\footnotetext{
${ }^{1}$ Laboratoire de Spectrométrie de Masse des Interactions et des Systèmes (LSMIS) UMR 7140 (Unistra-CNRS), Université de Strasbourg, France.

${ }^{2}$ Centre d'immunologie Pierre Fabre; Saint-Julien-en-Genevois, France.
}

\section{ABSTRACT:}

Capillary electrophoresis mass spectrometry coupling (CE-MS) is a growing technique in biopharmaceutics characterization. Assessment of monoclonal antibodies (mAbs) is well known at middle-up and bottom-up levels to obtain information about the sequence, posttranslational modifications (PTMs) and degradation products. Intact protein analysis is an actual challenge to be closer to the real protein structure. At this level, actual techniques are time consuming or cumbersome processes. In this work, a 20 minutes separation method has been developed to optimize characterization of intact mAbs. Thus, separation have been done on a positively-charged coated capillary (PEI) with optimized volatile background electrolyte (BGE) and sample buffer (SB). A sheathless interface allowed to hyphenate $\mathrm{CE}$ to a quadrupoletime-of-flight mass spectrometer (Q-TOF) which parameters has been tuned to improve the high masses detection and identification of intact mAbs. Three world-wide health authorities approved mAbs have been used to set up a rapid and ease of use method. Intact trastuzumab, rituximab and palivizumab isoforms have been partially separated with this method in less than 20 minutes under denaturing conditions. For each mAb, 2X-glycosylated and $1 X-$ glycosylated structures have been identified and separated. Concerning basic and acidic variants potential Iso-Asp modification and Asn deamidation have been observed. Accurate mass determination for high-mass molecular species remains a challenge, but the progress in intact $\mathrm{mAbs}$ separation appears very promising for biopharmaceutics characterization.

Keywords: Capillary Electrophoresis; Mass Spectrometry; Monoclonal Antibody; micro-variant separation, glycoform separation

Corresponding author: Emmanuelle Leize-Wagner: leize@unistra.fr 


\section{Introduction}

Monoclonal antibodies (mAbs) are tetrameric glycoproteins having a molecular mass of approximately $150 \mathrm{kDa}$, composed of two heavy chains and two light chains, inter-linked by several disulfide bonds, and having at least one conserved $\mathrm{N}$-glycosylation site located in the Fc domain ${ }^{1}$. mAbs were introduced for the treatment of various diseases in the late 1980 and they still represent the most rapidly growing category of therapeutic molecules today ${ }^{1-3}$. There are more than seventy-five mAbs approved by the US Food and Drug Administration (FDA) and the European Medicines Agency (EMA). mAb are particularly interesting because of their good therapeutic efficiency, favorable pharmacokinetic (PK) and pharmacodynamics (PD), and relatively low side-effects ${ }^{4}$.

Several separation-based methods both on liquid chromatography and electrophoresis are used for antibody characterization and homogeneity assessment ${ }^{5}$. These orthogonal analytical methods aim particularly to separate the antibody main isoform from micro-variants ${ }^{6}$. Micro-variants are commonly observed when mAbs are analyzed by charge-based separation techniques such as isoelectric focusing gel electrophoresis (IEF), capillary IEF (CIEF), imaged cIEF (icIEF), capillary zone electrophoresis (CZE) and ion exchange chromatography (CEX/ AEX) ${ }^{7}$. Many of the modifications leading to the formation of acidic and basic species have been identified as asparagines (Asn) deamidation, methionines oxidation, aspartic acid isomerization (Iso-Asp), cyclization of glutamic acid or glycosylation. mAb heterogeneity explains the difficulty of separation and identification of each isoform. In a theoretical way, millions of possibilities are available for only one mAb with plenty of weak masses difference between proteoforms. Most of these PTMs have been localized and characterized by different techniques as liquid chromatography-tandem mass spectrometry (LC-MS/MS) or capillary electrophoresis-tandem mass spectrometry coupling (CE-MS/MS), 8-10. LC-MS/MS and CE-MS/MS analysis, built on a classical bottom-up proteomics strategy, allowed to get a lot of information about mAbs proteoforms and their PTMs but workflows can induce some modifications or degradations of therapeutic proteins.

This last decade, separation of intact mAbs have been studied with several electrophoretic techniques such as CZE, 2D-CE-MS and CZE-MS ${ }^{11-14}$. He et al were precursor in the field of intact mAbs separation using CZE-UV. They developed a rapid method using $\varepsilon$-amino-caproic acid (EACA) and triethylenetetramine (TETA) as BGE, and hydroxypropylmethyl cellulose (HPMC) as dynamic neutral coating of the capillary ${ }^{11}{ }^{15}$. In 2015 Moritz et al validated this method by an inter-laboratory study allowing to consider it as the reference method for charge heterogeneity assessment of $\mathrm{mAbs}^{16}$. Other approach using UV detection was recently described by Goyon et al to set up a simpler CZE method to separate more acidic and basic variants for a dozen of commercial mAbs ${ }^{17}$. However, the composition of BGEs described in these studies are not compatible with ESI-MS detection due to high salt 
concentration and the presence of detergents and polymers. Since 2017, Neusüß's group developed an original 2D-CE-MS instrumentation allowing the separation of intact $\mathrm{mAbs}$ by CZE-CZE-MS and icIEFCZE-MS ${ }^{18,19}$. While the first CZE dimension enabling to use the same BGE condition as the reference CZE-UV method described by He et $a l^{15}$, the second dimension allowed the MS characterization by the use of BGEs compatible with ESI-MS detection. Joo $\beta$ et al illustrated their method for the detailed MS characterization of $\mathrm{mAbs}$ charge variants ${ }^{18}$. They highlighted glycosylated and deglycosylated variants and potential deamidation products for an intact antibody. While this approach appears very promising, CZE-CZE-MS setup needed heavy instrumental development and the overall analysis time is rather long. More recently, Belov et al developed a CZE-ESI-MS method to characterize one unknown IgG1 mAb by both middle-down and intact levels. At the intact level, CZE-ESI-MS analysis were performed under denaturing conditions and using a non-commercial positive coating (M7C4I). Baseline separation of the $2 \mathrm{X}$-glycosylatd, $1 \mathrm{X}$-glycosylated, and aglycosylated populations were obtained in nearly 30 minutes ${ }^{20}$.

In this report, we developed a 20 minutes CZE-ESI-MS method for the analysis of three well-known approved mAbs at the intact level. Experiments were performed with a commercial positively-charged capillary coating of polyethylenelmine (PEI) to avoid adsorption phenomenon. Acidic background electrolyte (BGE) and acidic methanol/water sample buffer have been optimized in order to obtain micro-variants separation of intact mAbs in less than 20 minutes. Three world-wide health authorities approved mAbs: rituximab (chlgG1, CHO), palivizumab (hzlgG1, SP2/0) and trastuzumab (hzlgG1, CHO), were selected for this study. Comparison with reference CZE-UV methods have been achieved allowing potential characterization of basic and acidic variant regions. Separation of charge variants arising from 2X-glycosylated, 1X-glycosylated pattern has been identified while potential Iso-aspartic acid isomerization (Iso-Asp) and asparagine deamidation have been observed as basic and acidic variants.

\section{Experimental}

\section{Chemicals}

Chemicals used were of analytical grade or high purity grade and purchased from Sigma-Aldrich (Saint Louis, MO, USA). Water used to prepare buffers and sample solutions was obtained using an ELGA purelab UHQ PS water purification system (Bucks, UK). mAbs were obtained as European Union pharmaceutical-grade drug product from their respective manufacturers. 
For intact mAbs analysis; trastuzumab, rituximab and palivizumab were desalted to remove all residual components of the storage solution. Samples were buffer exchanged with milliQ water three times on Amicon centrifugal filters with a $10 \mathrm{kDa}$ cut off (Merck, Darmstadt, Germany). Each centrifugation was made at $14^{\prime} 000 \mathrm{~g}$ speed during 20 min to claw back $30 \mu \mathrm{L}$ of mAbs at a concentration of $33.3 \mu \mathrm{M}$. Samples were led to a final concentration in protein of $6.7 \mu \mathrm{M}$ using the desired sample buffers.

\section{Capillary electrophoresis}

All of the CE experiments have been done on a CESI8000 capillary electrophoresis system from Sciex Separation (Brea, CA, USA). Bare fused-silica capillaries (total length $100 \mathrm{~cm} ; 30 \mu \mathrm{m}$ i.d.) with a porous end from Sciex Separation (Brea, CA, USA) were positively coated with a commercial PolyEthylenelmine (PEI) coating following the protocol provided by Sciex Separation. A second capillary (total length $80 \mathrm{~cm} ; 50 \mu \mathrm{m}$ i.d.) was used to complete the electric line of the separation system. Before each analysis, both capillaries were rinsed at 75 psi during 3 min with 3\% acetic acid BGE. The 32 Karat $^{\mathrm{TM}}$ (Sciex Separation) software was used for instrument control and data acquisition. Hydrodynamic injection (2 psi for $10 \mathrm{sec}$ ) corresponding to a volume of $3 \mathrm{~nL}(0.5 \%$ of the capillary length) was used to inject the sample.

\section{Mass spectrometry}

The CE system was hyphenated to a maXis 4G (Bruker, Bremen, Germany) by the sheathless interface. This MS instrument is equipped with a hybrid analyzer composed of hexapoles followed by a time-offlight (TOF) analyzer. Sample were run in denaturating conditions and analyzed in a $\mathrm{m} / \mathrm{z}$ range from 2500 to 5000 . The Otof control 3.4 software allowed to pilot the nano-ESI source and the settings were the following ones: nanoESI voltage $+1500 \mathrm{~V}$, dry gas $3 \mathrm{~L} / \mathrm{min}$, ion funnels set at values of 400 and 400 $\mathrm{Vpp}$, is CID energy at $190 \mathrm{eV}$ and source temperature at $150^{\circ} \mathrm{C}$. The data acquisition was made in positive mode.

\section{Data analysis}

MS data have been analyzed with the dissect mode of Data Analysis 4.2 software (Bruker, Bremen, Germany) with an internal $\mathrm{S} / \mathrm{N}$ threshold of 3 and a maximum of 10 overlapping compounds. Cut-off intensity of mass spectrum calculation was set at $0.1 \%$. After an automatic interpretation of the 
results, a manual validation has been performed on the results. MS spectra have been extracted from each peak represented on the BPE (Base Peak Electropherogram). Each profile has been selected and deconvoluted between 140 and $160 \mathrm{KDa}$ with the maximum entropy algorithm provided by Bruker's software to calculate the mass of the corresponding compounds. Automatic and manual results have been confronted to detect any mass modifications and get some additional information about the different identified compounds.

\section{Results and discussion}

In this work, major separation parameters have been selected and optimized based on the agreement of intact mAbs analysis and limits of CE-MS coupling. To avoid protein adsorption on the inner surface of the capillary due to negative charge surface of the silanol groups, bare fused silica capillaries have been previously modified with a covalent coating of PEI which confers positive charge surface of the inner capillary wall and then involves a reverse electro-osmotic flow under an electric field. Online CZE-ESI-MS coupling excluding the use of non-volatile salts ${ }^{11}$, effects of volatile background electrolyte (BGE), sample buffer and injection volume were optimized on the separation of intact trastuzumab. This mAb can be considered as reference material for CZE-ESI-MS method development due to the large number of reports describing the physicochemical properties of the protein $^{7}$.

\section{Evaluation of BGE and sample buffer component effect on method development}

The most frequently used BGEs in CZE-ESI-MS under denaturing conditions are acetic acid and formic acid because of their conductivity and their volatility allowing a good compatibility with ESI-MS detection ${ }^{20-23}$. In our study, BGEs consisted in acetic acid and formic acid at different concentrations from 1 to $10 \%(\mathrm{v} / \mathrm{v})$ and 1 to $5 \%(\mathrm{v} / \mathrm{v})$ respectively, were evaluated to keep a current intensity compatible with the separation and to avoid any degradation of capillaries. No separation has been observed with formic acid BGEs in the tested conditions. Concerning acetic acid, increasing concentration from 1 to $10 \%$ induced a time shift for the compounds migration (Figure 1 ) due to the increase of ionic strength. $1 \%$ and $10 \%$ showed only one peak meaning that no separation of mAbs isoforms can be observed. However, 3 and $5 \%$ acetic acid gave partial separation of mAbs with poor resolution. Indeed, obtained peaks were not thin and resolved and deconvolution spectra showed partial overlapping of different isoforms. However, $3 \%$ acetic acid BGE gave the most encouraging separation of intact mAbs and was conserved to the next optimization step. 

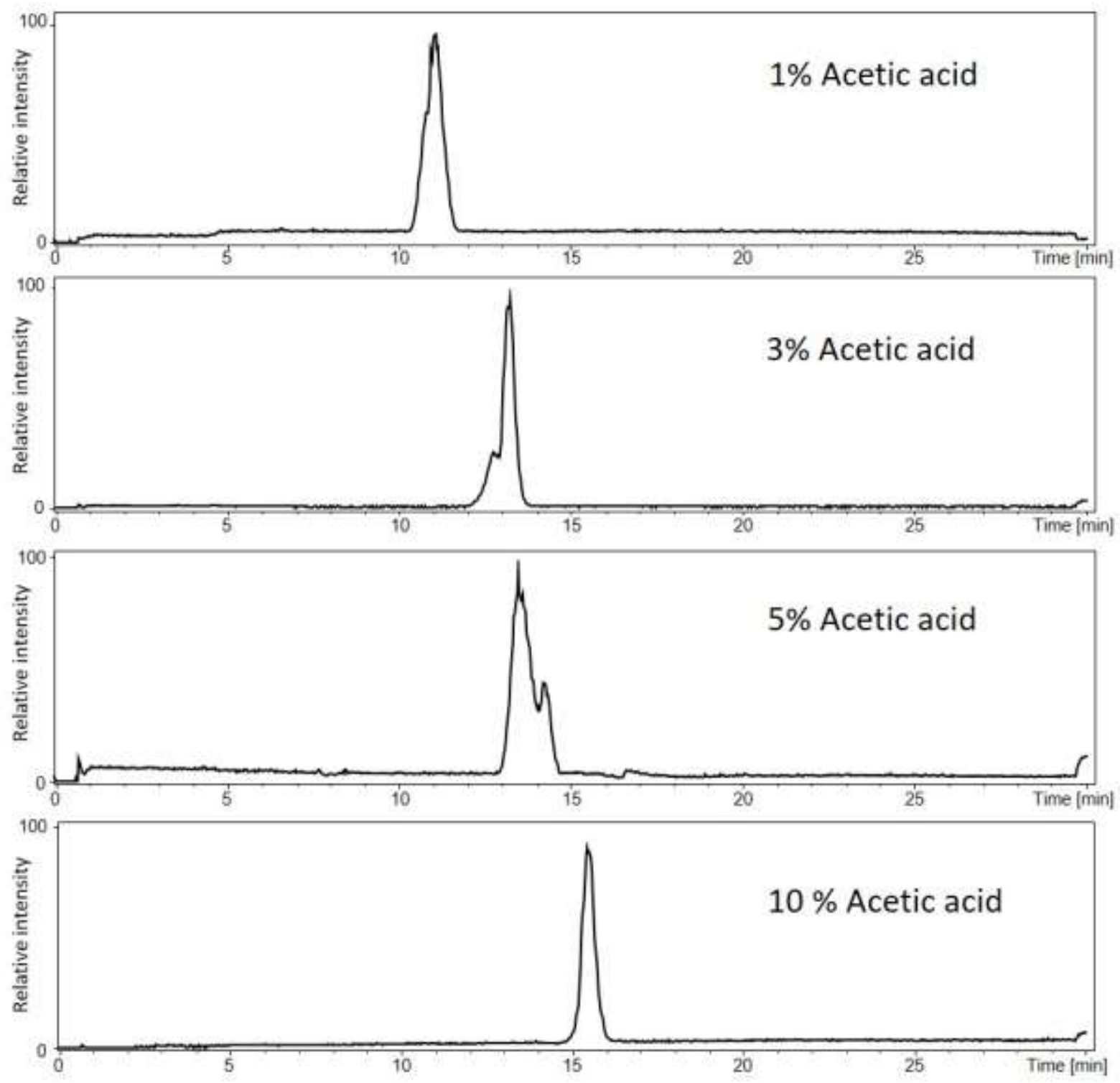

Figure 1. Effect of acetic acid concentration on the separation of trastuzumab charge variants. Acetic acid proportion was set up at $1 \%, 3 \%, 5 \%$ and $10 \%$.

Sample buffer is a parameter which can increase the performance of the separation, for instance due to difference of conductivity with BGE. The first part of this optimization step consisted in testing purified water, acetic acid and formic acid at different concentrations ( 1 to $10 \%(v / v)$ and 1 to $5 \%$ $(\mathrm{v} / \mathrm{v})$ respectively) as sample buffer. Obtained results with purified water and acetic acid showed no 178 increase of resolution in mAbs separation. Only formic acid gave better resolution partly due to the 179 sharpness of the peaks, however, no tremendous increase of separation has been observed. Nevertheless, $1 \%$ formic acid has been selected for the following steps. Based on the work of Schwer et $a /$ who have calculated the influence on the electroosmotic velocity of adding organic solvent to the electrolytes ${ }^{24}$, we assessed the addition of methanol at different ratio $(10$ to $50 \%(\mathrm{v} / \mathrm{v}))$ in the $1 \%$ formic 
acid sample buffer (Figure 2). Up to $30 \%$ Methanol, a partial separation was obtained while a total loss of resolution was observed for ratio above $30 \%$. Trastuzumab separation performed with $30 \%$ methanol, $1 \%$ formic acid sample buffer exhibited three peaks obtained in less than 15 minutes (RSD $<3 \%$ on migration times $(n=10))$. Other organic solvents have been investigated as acetonitrile and isopropanol in the same proportions, however only methanol has given some good results. Sample injection volume has also been studied to subvert a capillary overloading known to affect the separation of compounds. Trials were done from 1 to $20 \mathrm{~nL}$ corresponding to 7 to $350 \mathrm{fmol}$ of mAbs. Better results were obtained by injecting $3 \mathrm{~nL}(20 \mathrm{fmol})$ of trastuzumab in the PEl coated capillary.
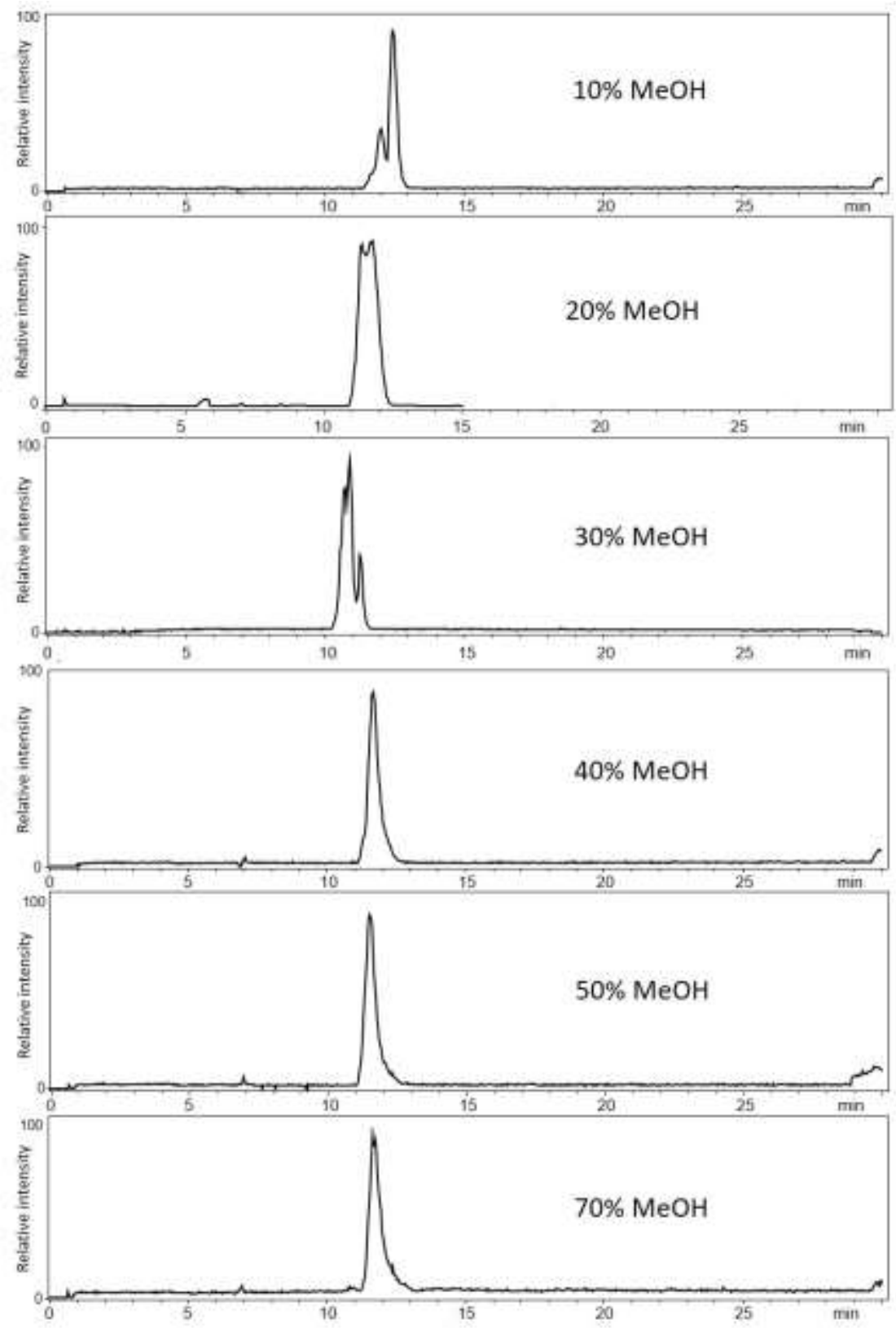

Figure 2. Effect of methanol concentration in the sample buffer for the separation of trastuzumab charge variants. Methanol proportion was set up at $10 \%, 20 \%, 30 \%, 40 \%, 50 \%$ and $70 \%$ on $1 \%$ formic acid (v/v). The BGE contained $3 \%$ acetic acid. 


\section{CZE-ESI-MS characterization of intact mAbs}

Since 2010, a some reports described CZE-based methods for the characterization of mAbs charges variants for the quality control of biopharmaceutical companies in terms of product heterogeneity ${ }^{11-14,18}$. To date, two reports from Ruesch's group represents a reference for the analysis of mAbs using CZE with a UV detection ${ }^{11,15}$. Nevertheless, no structural information can be detailed for the basic and/or acidic variants. In our study, optimized CZE-ESI-MS condition developed on trastuzumab sample has been assessed on two other well-known IgG1 mAbs: rituximab and palivizumab. Obtained results for these three mAbs are showed in Figure 3. Due to the reverse electroosmotic mode involved by the use of PEI coated capillary, electropherograms of each mAbs exhibited three peaks distributed as acidic variants in the first peak, the main variant in the second peak and basic variants in the third peak. Despite the worst resolution obtained in CZE-ESI-MS as compared to reference CZE-UV method which can be easily understood by the difference of BGE condition ( $\mathrm{pH} 5.7$, presence of TETA...) and the inverted profiles of the three regions due to reverse mode, obtained CZE-ESI-MS electropherograms fitted well with reference CZE-UV electropherograms. Each mAbs following quite well similar behavior in appearance, trastuzumab has been chosen to illustrate globally the obtained MS results. However, differences will be detailed for rituximab and palituzumab. For each peak, deconvoluted mass spectra exhibited the classical glycoform pattern of trastuzumab (Figure 4). Focused on the three highest abundance glycoforms of the main peak, average masses of $148,057 \pm 3 \mathrm{Da}, 148,218 \pm 2 \mathrm{Da}$, and 148,380 \pm 3 Da were measured corresponding to GOF/GOF, GOF/G1F, and G1F/G1F glycoforms respectively. Mass delta of around 162 Da between the three glycoforms agreed with the theoretical mass of a galactose moiety. However, glycan structure with the addition of galactose moieties does not induce a change in net charge ${ }^{25-27}$. Gahoual and coworkers demonstrated that particular glycopeptides having a difference of one galactose could be baseline separated ${ }^{8}$ whereas Redman et al did not observe mobility shifts between intact mAb glycoforms due to the low impact of $162 \mathrm{Da}$ on the global mass of the mAb $(\approx 0.1 \%)^{23}$. Our work follows Redman et al observations with no separation of intact mAb variants due to differences of $2 \mathrm{X}$ glycosylated forms. However, in each cases, manual analysis of raw data enabled to characterize $1 \mathrm{X}-$ glycosylated forms overlapping with the last peak meaning that separation between 2X-glycosylated and 1X-glyxosylated forms are obtained for the three mAbs. This confirms the results recently described by Belov et al on an unknown mAbs ${ }^{20}$. 


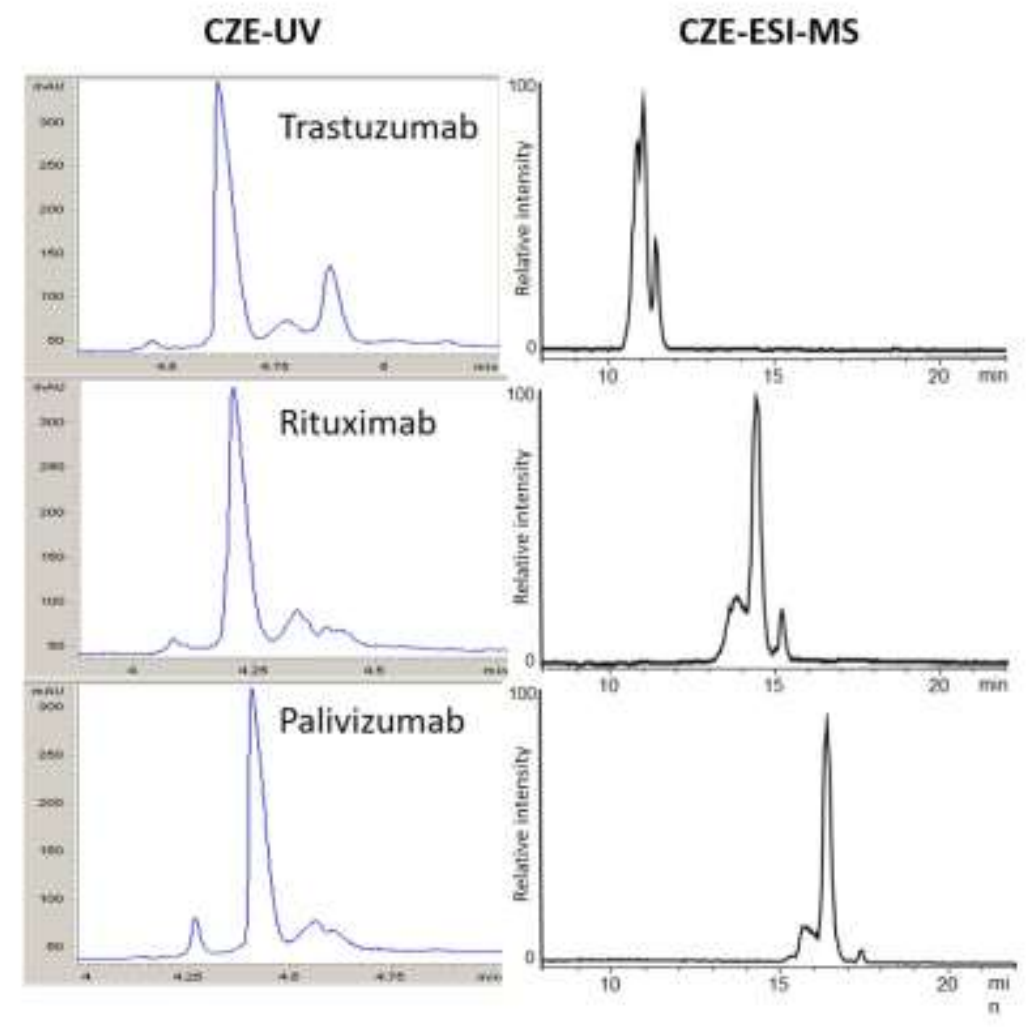

Figure 3. CZE-ESI-MS separation of intact tratuzumab, rituximab and palivizumab obtained with the optimized settings and CZE-UV profiles according to the methodology of He et al ${ }^{15}$. Reprinted from 17 . Copyright (2018), Wiley.

For each mAb, peaks corresponding to possible acidic or basic variants have been deconvoluted. Concerning basic variant, a mass difference of -1 to -2 Da compared with the main variant was observed for the three mAbs $(148,217 \pm 4 \mathrm{Da}$ as compared to $148,218 \pm 3 \mathrm{Da}$ for trastuzumab), while a mass difference of -2 Da was observed only for trastuzumab $(148,216 \pm 4 \mathrm{Da})$ for acidic variant. As already described in the literature, the most important antibody basic charge variants are iso-Asp modification, C-terminal Lys truncation, aglycosylation, incomplete cyclization of the N-terminal glutamine (GIn) to pyroGlu or methionine oxidation; whereas sialylation, asparagine deamidation, glycation, cysteinylation are the most commonly observed acidic variants ${ }^{28}$. Trastuzumab possesses several Asp and Asn residues in its amino acid sequence which can be potentially iso-Asp or deamidated $^{10,29}$. Even if the standard deviation of mass measurement less than 4 Da doesn't allow to conclude without any ambiguity on the exact nature of the modification, we can explain the mass shift of less than $2 \mathrm{Da}$ by potential iso-Asp modification for basic charge variants and potential Asn deamidation for acidic charge variants. These results are in agreement with the literature obtained for 
247 the characterization of trastuzumab in a 2D-CZE-MS coupling ${ }^{18}$. However, while rituximab and 248 palivizumab also possess potential modified Asn, no acidic variant characterization has been observed 249 using our CZE-ESI-MS condition. Indeed, for rituximab, third peak deconvoluted mass spectrum gives any masses corresponding to intact mAbs or degradation products but an unknown impurity, and for palivizumab, deconvoluted mass spectra fitted with $1 \mathrm{X}$-glycoform pattern. These results can potentially ask the real nature of acidic variants found by the CZE-UV reference methods for these two mAbs and highlights the constant need of method development with MS detection to obtained structural characterization.

\section{Conclusion}

In this study, trastuzumab, rituximab and palivizumab were analyzed at the intact level by CZEESI-MS. A rapid separation method has been developed to characterize these commercial mAbs under denaturating condition. A PEI positive coating has been set up to avoid protein adsorption on the inner surface of the capillary. Separation has been performed in 3\% acetic acid BGE at $30 \mathrm{kV}$ and sample buffer has been optimized to $30 \%$ methanol, $1 \%$ formic acid with each mAbs to a final concentration of $6.7 \mu \mathrm{M}$. CZE-ESI-MS analysis of these three mAbs showed partial separation obtained in less than 20 minutes allowing identification of mAbs isoforms. As a first result, CZE-ESI-MS electropherograms fitted quite well with reference CZE-UV electropherograms allowing a potential characterization of the basic and acidic variant regions. For each mAbs, 2X-glycosylated and $1 \mathrm{X}$-glycosylated structures has been identified and separated. Concerning basic and acidic variants, minor differences between 0 to 2 Da have been observed suggesting potential Iso-Asp modification and Asn deamidation. However, mass precision didn't allow to conclude without any ambiguity on the nature of these modifications. Accurate mass determination for high-mass molecular species remains a challenge, but the progress in intact mAbs separation appears very promising and could be recognized as an additional step in biopharmaceutics characterization.

272 
Trastuzumab
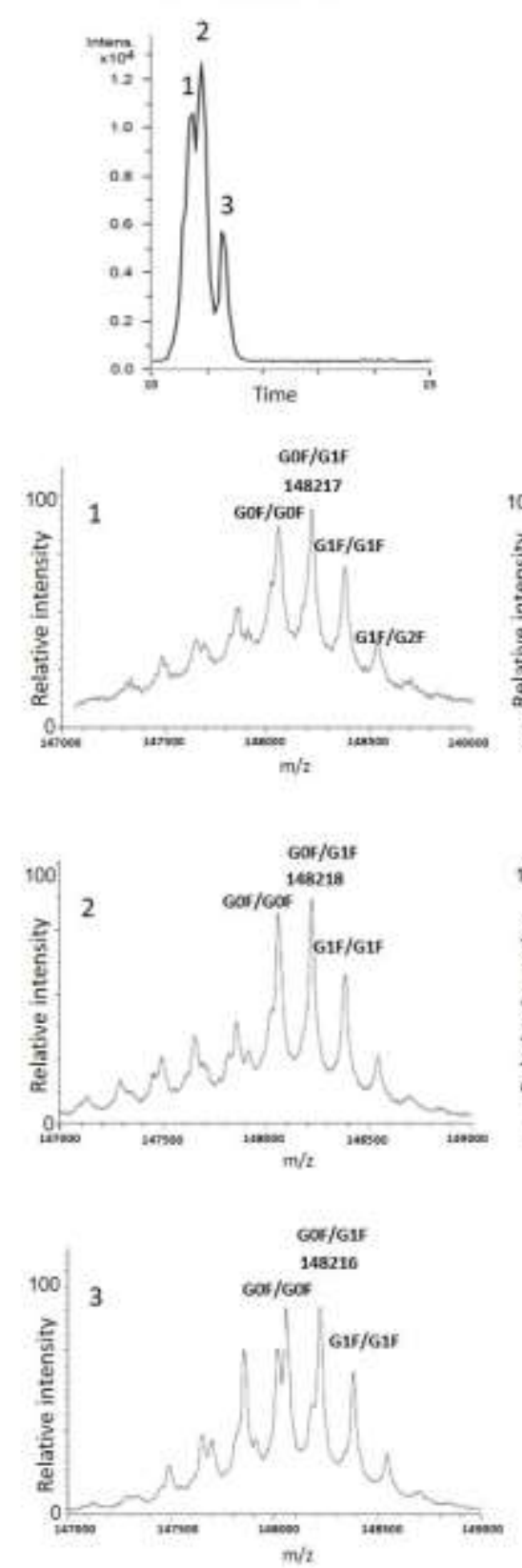

Rituximab
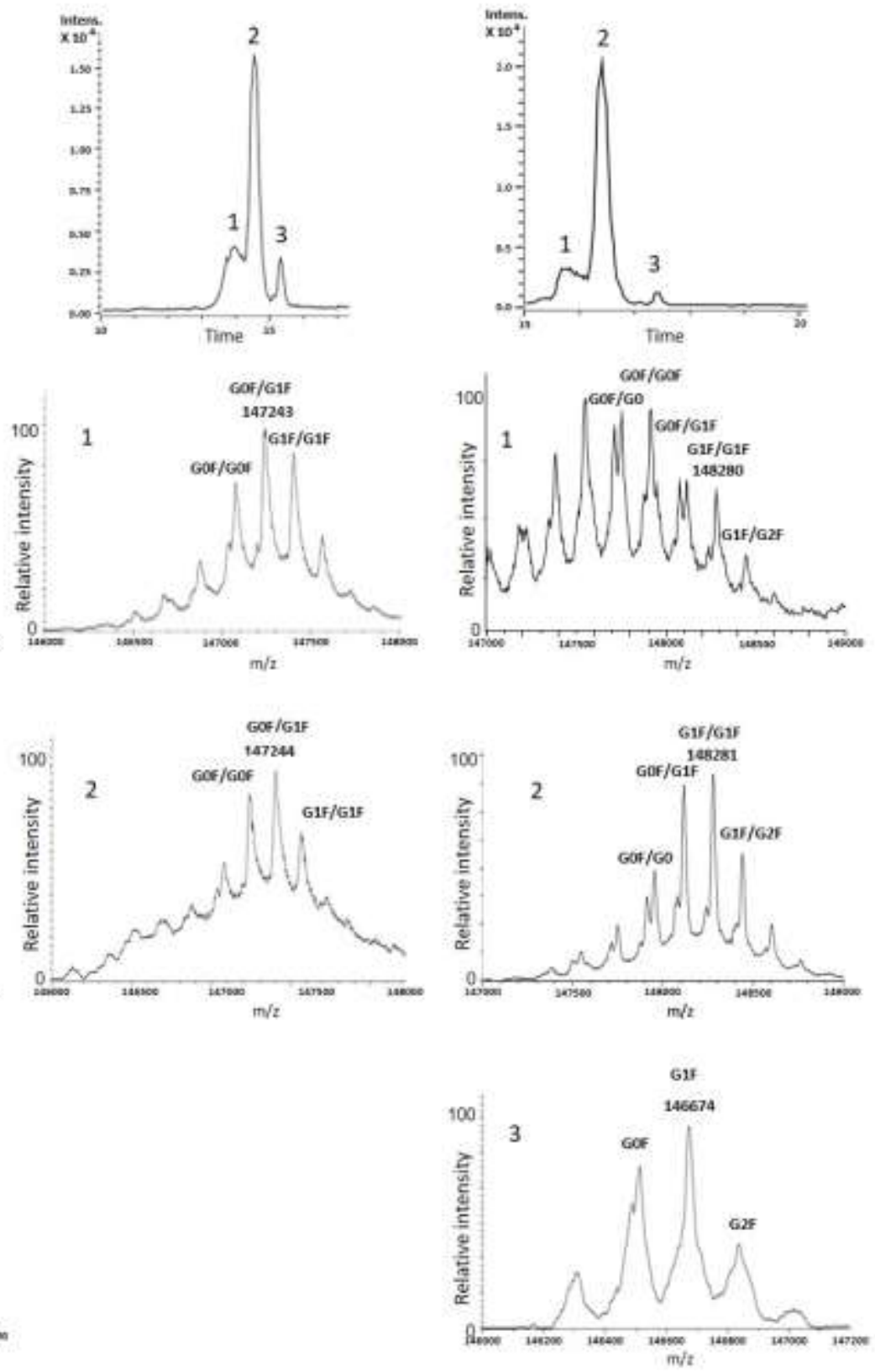

279 Figure 4. CZE-ESI-MS separation of intact tratuzumab, rituximab and palivizumab obtained with the 280 optimized settings. For each mAbs, deconvoluted mass spectra was performed for the basic variant 281 (1), main variant (2), and acidic variant (3)

\section{Acknowledgments}

284 Authors would like to thank Sciex and Bruker Daltonics for the fruitful collaboration and the support. The authors would like also to express their gratitude to Dr. E. Wagner-Rousset, , MC. Janin-Bussat and O. Colas (Centre d'Immunologie Pierre Fabre, St Julien en Genevois, France) for helpful discussions 
287 around antibody structural characterization by LC-MS. This work was supported by the CNRS (UMR 288 7140), the University of Strasbourg, and a doctoral fellowship from Bruker Daltonics and the General 289 Council of Region Grand Est to JG. 
291 1. Berger M, Shankar V and Vafai A. Therapeutic Applications of Monoclonal Antibodies. The American Journal of the Medical Sciences. 2002; 324: 14-30.

2. Reichert JM. Marketed therapeutic antibodies compendium. mAbs. 2012; 4: 413-5.

3. Beck A, Wurch T, Bailly $C$ and Corvaia N. Strategies and challenges for the next generation of therapeutic antibodies. Nat Rev Immunol. 2010; 10: 345-52.

4. Wang W, Singh S, Zeng DL, King K and Nema S. Antibody structure, instability, and formulation. Journal of Pharmaceutical Sciences. 2007; 96: 1-26.

5. Fekete S, Guillarme D, Sandra P and Sandra K. Chromatographic, Electrophoretic, and Mass Spectrometric Methods for the Analytical Characterization of Protein Biopharmaceuticals. Analytical Chemistry. 2016; 88: 480-507.

6. Doneanu CE, Xenopoulos A, Fadgen K, et al. Analysis of host-cell proteins in biotherapeutic proteins by comprehensive online two-dimensional liquid chromatography/mass spectrometry. Mabs. 2012; 4: 24-44.

7. Gahoual R, Beck A, Leize-Wagner E and François Y-N. Cutting-edge capillary electrophoresis characterization of monoclonal antibodies and related products. Journal of Chromatography B. 2016; 1032: 61-78.

8. Gahoual R, Busnel J-M, Beck A, François $\mathrm{Y}-\mathrm{N}$ and Leize-Wagner E. Full Antibody Primary Structure and Microvariant Characterization in a Single Injection Using Transient Isotachophoresis and Sheathless Capillary Electrophoresis-Tandem Mass Spectrometry. Analytical Chemistry. 2014; 86: 9074-81.

9. Said N, Gahoual R, Kuhn L, Beck A, François Y-N and Leize-Wagner E. Structural characterization of antibody drug conjugate by a combination of intact, middle-up and bottom-up techniques using sheathless capillary electrophoresis - Tandem mass spectrometry as nanoESI infusion platform and separation method. Analytica Chimica Acta. 2016; 918: 50-9.

10. Gahoual R, Beck A, François $\mathrm{Y}-\mathrm{N}$ and Leize-Wagner $\mathrm{E}$. Independent highly sensitive characterization of asparagine deamidation and aspartic acid isomerization by sheathless CZE-ESIMS/MS. Journal of Mass Spectrometry. 2016; 51: 150-8.

11. He Y, Lacher NA, Hou W, et al. Analysis of Identity, Charge Variants, and Disulfide Isomers of Monoclonal Antibodies with Capillary Zone Electrophoresis in an Uncoated Capillary Column. Analytical Chemistry. 2010; 82: 3222-30.

12. Schlecht J, Jooß $\mathrm{K}$ and Neusüß C. Two-dimensional capillary electrophoresis-mass spectrometry (CE-CE-MS): coupling MS-interfering capillary electromigration methods with mass spectrometry. Analytical and Bioanalytical Chemistry. 2018.

13. Han M, Rock BM, Pearson JT and Rock DA. Intact mass analysis of monoclonal antibodies by capillary electrophoresis-Mass spectrometry. Journal of Chromatography B. 2016; 1011: 24-32.

14. Belov AM, Viner R, Santos MR, et al. Analysis of Proteins, Protein Complexes, and Organellar Proteomes Using Sheathless Capillary Zone Electrophoresis - Native Mass Spectrometry. Journal of The American Society for Mass Spectrometry. 2017; 28: 2614-34.

15. He Y, Isele $\mathrm{C}$, Hou W and Ruesch M. Rapid analysis of charge variants of monoclonal antibodies with capillary zone electrophoresis in dynamically coated fused-silica capillary. Journal of Separation Science. 2011; 34: 548-55.

16. Moritz B, Schnaible V, Kiessig S, et al. Evaluation of capillary zone electrophoresis for charge heterogeneity testing of monoclonal antibodies. Journal of Chromatography B. 2015; 983-984: 10110.

17. Goyon A, Francois YN, Colas O, Beck A, Veuthey JL and Guillarme D. High-resolution separation of monoclonal antibodies mixtures and their charge variants by an alternative and generic CZE method. Electrophresis. 2018; 0.

18. Jooß K, Hühner J, Kiessig S, Moritz B and Neusüß C. Two-dimensional capillary zone electrophoresis-mass spectrometry for the characterization of intact monoclonal antibody charge variants, including deamidation products. Analytical and Bioanalytical Chemistry. 2017; 409: 6057-67. 
19. Montealegre $C$ and Neusüß C. Coupling imaged capillary isoelectric focusing with mass spectrometry using a nanoliter valve. ELECTROPHORESIS. 2018; 39: 1151-4.

20. Belov AM, Li L, Roberto S, et al. Complementary middle-down and intact monoclonal antibody proteoform characterization by capillary zone electrophoresis - mass spectrometry. Electrophroresis. 2018; 0 .

21. Faserl K, Sarg B, Kremser L and Lindner H. Optimization and Evaluation of a Sheathless Capillary Electrophoresis-Electrospray lonization Mass Spectrometry Platform for Peptide Analysis: Comparison to Liquid Chromatography-Electrospray lonization Mass Spectrometry. Analytical Chemistry. 2011; 83: 7297-305.

22. Haselberg R, Harmsen S, Dolman MEM, de Jong GJ, Kok RJ and Somsen GW. Characterization of drug-lysozyme conjugates by sheathless capillary electrophoresis-time-of-flight mass spectrometry. Analytica Chimica Acta. 2011; 698: 77-83.

23. Redman EA, Mellors JS, Starkey JA and Ramsey JM. Characterization of Intact Antibody Drug Conjugate Variants Using Microfluidic Capillary Electrophoresis-Mass Spectrometry. Analytical Chemistry. 2016; 88: 2220-6.

24. Schwer $C$ and Lottspeich F. ANALYTICAL AND MICROPREPARATIVE SEPARATION OF PEPTIDES BY CAPILLARY ZONE ELECTROPHORESIS USING DISCONTINUOUS BUFFER SYSTEMS. Journal of Chromatography. 1992; 623: 345-55.

25. Biacchi M, Gahoual R, Said N, Beck A, Leize-Wagner E and François Y-N. Glycoform Separation and Characterization of Cetuximab Variants by Middle-up Off-Line Capillary Zone ElectrophoresisUV/Electrospray lonization-MS. Analytical Chemistry. 2015; 87: 6240-50.

26. Raju TS and Scallon BJ. Glycosylation in the Fc domain of IgG increases resistance to proteolytic cleavage by papain. Biochemical and Biophysical Research Communications. 2006; 341: 797-803.

27. Liu HC, Gaza-Bulseco G, Faldu D, Chumsae C and Sun J. Heterogeneity of monoclonal antibodies. Journal of Pharmaceutical Sciences. 2008; 97: 2426-47.

28. Du Y, Walsh A, Ehrick R, Xu W, May K and Liu H. Chromatographic analysis of the acidic and basic species of recombinant monoclonal antibodies. $m A$ Abs. 2012; 4: 578-85.

29. Gahoual R, Burr A, Busnel JM, et al. Rapid and multi-level characterization of trastuzumab using sheathless capillary electrophoresis-tandem mass spectrometry. Mabs. 2013; 5: 479-90. 